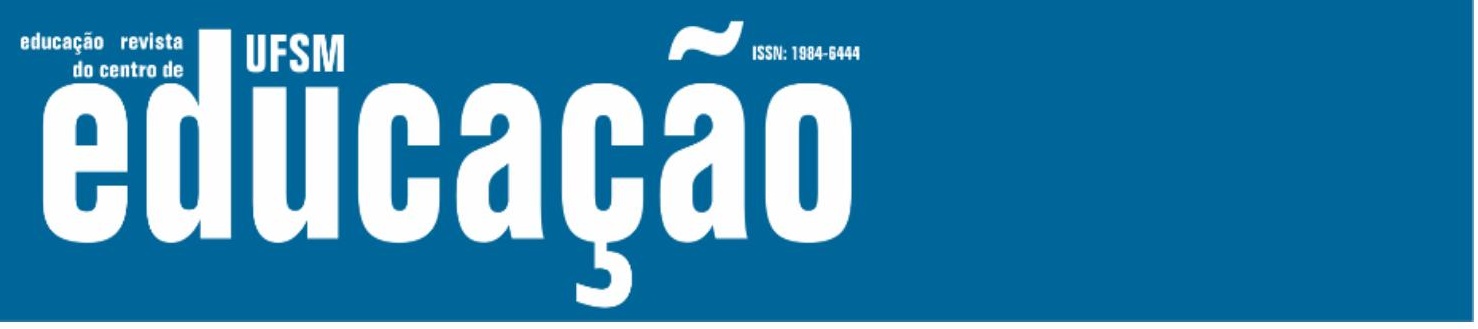

ISSN: 1984-6444 | http://dx.doi.org/10.5902/198464434406

\title{
Aspectos a serem considerados em investigações a respeito do movimento de constituição da Identidade Profissional de professores que ensinam matemática
}

Aspects to be considered in studies on the movement of constitution of Professional Identity in educators who teach mathematics

Enio Freire de Paula

Professor doutor do Instituto Federal de Educação, Ciência e Tecnologia de São Paulo, Campus de Presidente Epitácio, São Paulo, Brasil.

eniodepaula@ifsp.edu.br - http://orcid.org/0000-0003-0395-4689

Márcia Cristina de Costa Trindade Cyrino

Professora Associada da Universidade Estadual de Londrina. Londrina, Paraná, Brasil. marciacyrino@uel.br - http://orcid.org/0000-0003-4276-8395

Recebido em 22 de agosto de 2018

Aprovado em 16 de julho de 2019

Publicado em 29 de março de 2020

\section{RESUMO}

O objetivo do presente artigo é discutir aspectos a serem considerados em investigações a respeito do movimento de constituição da Identidade Profissional (IP) de professores que ensinam matemática (PEM). Para tanto, analisaram-se e problematizaram-se aspectos associados: (i) aos processos de constituição da IP de PEM; (ii) aos contextos investigativos; e (iii) às perspectivas epistemológicas de IP de PEM, identificados em investigações a respeito da IP de PEM no decorrer da construção e da análise de dois corpora, tendo como recorte temporal, o período de 2006-2016. Os resultados evidenciam que a complexidade, a dinamicidade, a temporalidade e a experiencialidade são aspectos importantes a serem levados em conta pelas pesquisas que se propõem a investigar os processos de constituição da IP de PEM. Quanto ao contexto investigativo, ações envolvendo PEM já atuantes na Educação Básica, futuros professores e docentes universitários em ambientes grupais fomentam a integração entre os participantes e a valorização de suas práticas profissionais. Em relação às perspectivas epistemológicas, é imprescindível reconhecer que as escolhas do pesquisador influenciam todo o processo de análise. Nesse sentido, problematizar a IP nas investigações em uma perspectiva formativa pode fomentar a valorização das práticas profissionais de (futuros) PEM e evidenciar momentos de tensão/fragilidade potencializadores desses processos de formação necessários para o movimento de constituição da IP.

Palavras-chave: Identidade profissional de Professores que ensinam matemática; Formação de professores; Educação Matemática. 


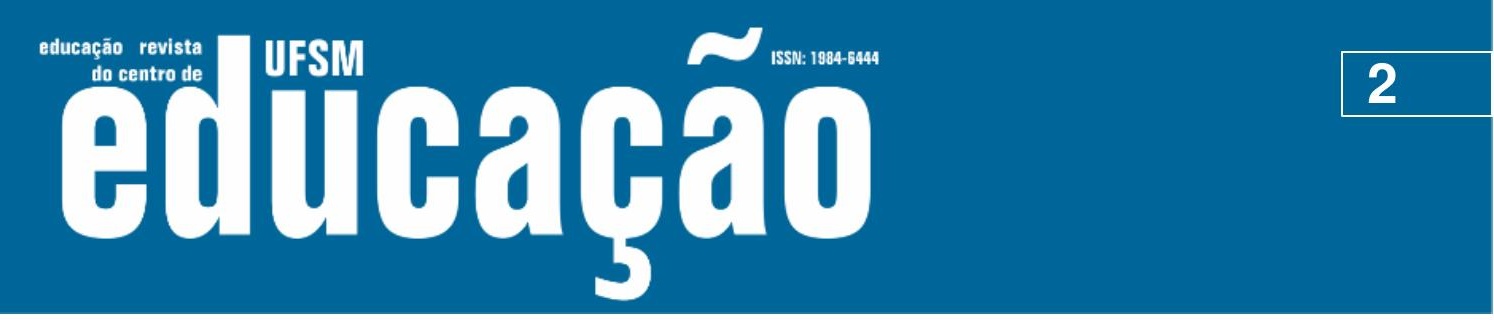

ISSN: 1984-6444 | http://dx.doi.org/10.5902/1984644434406

\section{ABSTRACT}

This article seeks to discuss some aspects to be considered during the movement of constitution of Professional Identity (PI) in educators who teach Mathematics. To do so, we analyzed and discussed some aspects linked (i) to the processes of constitution of PI in educators who teach Mathematics, (ii) to investigative contexts and (iii) to the epistemological perspectives of PI in educators who teach Mathematics, identified in studies on $\mathrm{Pl}$ during the construction/analysis of two corpora. The time frame approached in this study is 2006-2016. Results show that complexity, dynamics, temporality and experience are important aspects that should be taken into consideration in studies that investigate the processes of constitution of PI in educators who teach Mathematics. The investigative context involved educators who teach Mathematics in Basic Education levels, future teachers and university professors in group environments to foster integration among participants and to value their professional practices. When it comes to epistemological perspectives, it is vital to recognize that a researcher's choices influence the whole process of analysis. In this sense, discussing $\mathrm{PI}$ in studies through a formative perspective may foster the appreciation of (future) educators who teach mathematics' professional practice and evidence moments of tension/fragility that intensify the processes of formation, which are necessary to the movement of constitution of $\mathrm{PI}$.

Keywords: Professional identity of Teacher who teaches Mathematics; Teacher Education; Mathematics Education.

\section{Introdução}

Em estudos recentes, temos assumido a caracterização proposta por Cyrino (2016a, 2017), no qual a Identidade Profissional (IP) de professores que ensinam matemática (PEM) é constituída em um movimento que "se dá tendo vista um conjunto de crenças e concepções interconectadas ao autoconhecimento e aos conhecimentos a respeito de sua profissão, associado à autonomia (vulnerabilidade e sentido de agência) e ao compromisso político" (CYRINO, 2017, p. 704). Essa escolha está intimamente associada a dois fatores, interligados, que merecem destaque.

O primeiro deles é fruto de nossa participação no Grupo de Estudo e Pesquisa Grupo de Estudo e Pesquisa sobre Formação de Professores que Ensinam Matemática - Gepefopem ${ }^{1}$-, cujo histórico da produção de seus membros tem evidenciado a necessidade e a preocupação de investigar diferentes contextos de formação de professores (inicial e continuada), tais como: estágio curricular 


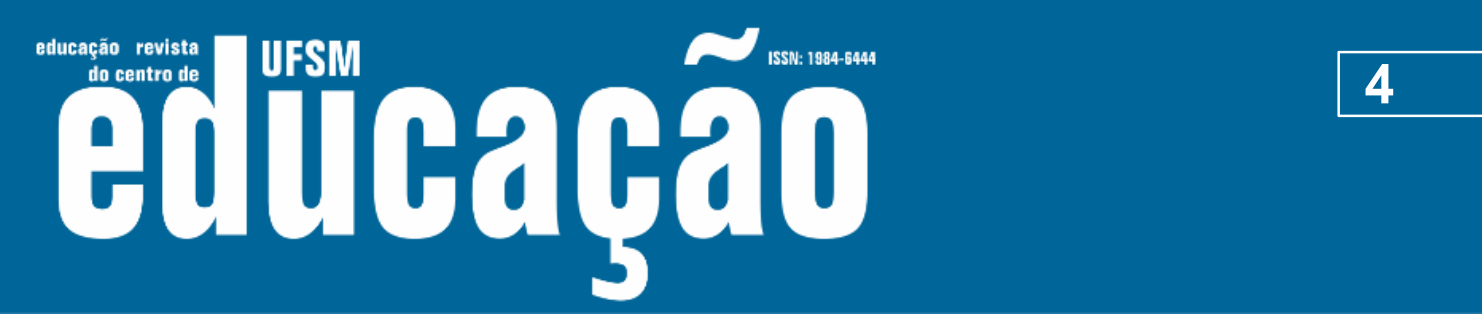

ISSN: 1984-6444 | http://dx.doi.org/10.5902/1984644434406

Sendo assim, no presente artigo discutimos aspectos a serem levados em conta em investigações a respeito do movimento de constituição da IP de PEM. Para tanto, analisamos e problematizamos investigações sobre o PEM presentes em dois corpora. Na sequência, apresentamos como eles foram constituídos e os aspectos associados aos processos de constituição da IP de PEM, aos contextos investigativos e às perspectivas epistemológicas de IP de PEM problematizados a partir desses estudos. Prosseguimos com o entrelaçar desses elementos e, por fim, nas considerações finais, sinalizamos questionamentos para futuras investigações nesse campo.

\section{Os corpora}

O No Quadro 1, apresentamos os estudos a respeito da IP de PEM, publicados entre 2006 - 2016, que compõem os corpora, quais sejam:

a) Corpus 1: 24 dissertações e teses brasileiras oriundas de Programas de Pósgraduação stricto sensu das áreas de Educação e Ensino.

b) Corpus 2: 23 artigos publicados em periódicos ${ }^{4}$ representativos do campo investigativo da Educação Matemática.

Quadro 1 - Apresentação e constituição dos corpora analisados

\begin{tabular}{c|l}
\hline Corpora & \multicolumn{1}{|c}{ Estudos integrantes dos corpora analisados } \\
\hline $\begin{array}{c}\text { Corpus 1 (analisados por } \\
\text { De Paula e Cyrino, }\end{array}$ & Zanini (2006), Batista Neto (2007), Beranger (2007), Chauvet \\
2017,2018a, 2018b) & (2008), Matheus (2008), Paz (2008), Sousa (2009), Souza \\
& (2009), Guidini (2010), Junqueira (2010), Moraes (2010), Beline \\
& (2013), Levy (2013), Teixeira (2013), Garcia (2014), Vieira \\
& (2014), Oliveira (2015), Barbato (2016), Severino (2016) e Kuhn \\
& (2016) \\
\hline Corpus 2 (analisados por & Lloyd (2006), Hodgen Askew (2007), Goos e Bennison (2008), \\
De Paula e Cyrino, 2018c) & Owens (2008), Boylan (2010), Gonçalves Costa e Pamplona \\
& (2011), Bjuland, Cestari e Borgersen (2012), Hobbs (2012), \\
& Kaasila, Hannula e Laine (2012), Nolan (2012), Ticknor (2012), \\
& Brown et al (2013), Gellert, Espinoza e Barbé (2013), Hossain, \\
& Mendrick e Adler (2013), Sanhueza, Penalva e Friz (2013), \\
& Gonçalves e De Carvalho (2014), Lutovac e Kaasila (2014), Van \\
& Putten, Stols e Howie (2014), Benninson (2015), Skog e \\
& Anderson (2015), Teixeira e Cyrino (2015), Cyrino (2016a) e Paz \\
& e Frade (2016)
\end{tabular}

Fonte: Elaborado pelos autores. Legenda das cores dos estudos: teses em vermelho, mestrados acadêmicos em verde e mestrados profissionais em azul. 


\section{Aillbapẫ \\ 3}

ISSN: 1984-6444 | http://dx.doi.org/10.5902/1984644434406

definição formal, finita, com limites bem (pré)determinados é inviável, posto que a IP de PEM está em constante movimento. Assim, não há critérios de precedência, superação ou relevância, entre esses quatro aspectos (CDTE), mas, sim, vínculos de proximidades e coalisões, cujas fronteiras são tênues. Uma representação possível, nessa ocasião, seria uma conexão, como ilustra a Figura 1.

Figura 1 - Conexão dos aspectos dos processos de constituição da IP de PEM

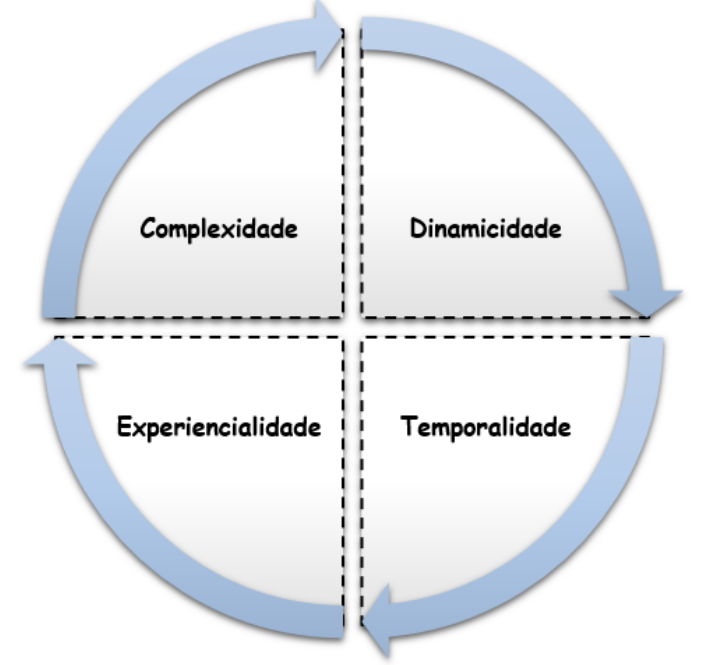

Fonte: Elaborado pelos autores

CDTE é o que qualifica o movimento. A IP de PEM é complexa, pois envolve múltiplos e influentes fatores; é dinâmica, pois as inter-relações entre esses fatores ocasionam mudanças - às vezes intempestivas; é temporal, pois o comportamento desses fatores é imprevisível; e é experiencial, pois cada indivíduo - pensando na relação indivíduo/sociedade - vê, interpreta, é atingido e reage a esses fatores de modo distinto.

\section{Aspectos caracterizadores dos contextos investigativos}

Lessard-Hebert, Goyette, Boutin (1994), que defendem um modelo articulado em quatro polos - epistemológico, teórico, morfológico e técnico - como um sistema interativo constituinte dos processos investigativos, foi nossa lente teórica ao analisarmos o Corpus 1 (DE PAULA; CYRINO, 2018c) especificamente os polos epistemológico e teórico. Apresentamos na Figura 2, uma síntese das principais 


\section{- Tusm Autlathat}

ISSN: 1984-6444 | http://dx.doi.org/10.5902/1984644434406

em Matemática - nas investigações é expressivo: entre eles os profissionais das áreas de Engenharia e Pedagogia, sendo que os (futuros) pedagogos são o grupo de maior destaque.

$\checkmark$ As experiências formativas ocorridas nesses contextos fomentam a integração entre os participantes e a valorização de suas práticas profissionais.

$\checkmark$ Os pesquisadores interessados em discutir a IP de PEM no contexto da formação inicial assumem a carência de estudos nessa direção como uma das dificuldades do processo de levantamento de seus referenciais teóricos.

$\checkmark$ As ações pertinentes ao desenvolvimento do Estágio Curricular Supervisionado (ECS) na licenciatura em Matemática são potencializadoras dos processos de constituição da IP de PEM.

$\checkmark$ Os estudos evidenciam, intencionalmente ou não, momentos de tensão/fragilidade de PEM, seja por identificação ou por (com)vivência.

Tratando especificamente do Corpus 1 destacamos: (i) as dificuldades nos processos de escolha/apresentação/discussão de referenciais teóricos diretamente relacionados à IP de PEM; (ii) os estudos em que os elementos do polo técnico deveriam ser evidenciados e problematizados com maior ênfase; e (iii) a ausência, em um número significativo dos estudos, de caracterizações a respeito da compreensão do(a) autor(a), ou da visão compartilhada por ele(a) sobre a concepção de IP de PEM.

Do Corpus 2 se destacam: (i) elementos dos polos epistemológico e teórico (com destaque para o quadro referencial a respeito da IP claramente delineado); (ii) maior número de pesquisas em que a constituição da IP de PEM é vinculada à problematização da aprendizagem de temas matemáticos específicos; e (iii) estabelecimento de relações entre a IP, as práticas profissionais e os modos de representatividade da IP.

Essas peculiaridades evidenciam as influências das escolhas teóricas e epistemológicas no encaminhamento metodológico das investigações.

Os estudos integrantes do Corpus 2 (no qual constam pesquisas nacionais e internacionais) avançam em relação ao Corpus 1 (de dissertações e teses) pelo cuidado dedicado aos elementos do modelo quadripolar. Inferimos que esse fator seja 


\section{T usm

ISSN: 1984-6444 | http://dx.doi.org/10.5902/1984644434406

decorrente da natureza morfológica da apresentação dos trabalhos. Nos estudos do Corpus 2, é perceptível a preocupação com a clareza e a explicitação dos elementos demarcadores dos polos epistemológico, teórico e técnico. Contribuem para essa preocupação os limites (característicos) impostos aos pesquisadores para esse tipo de publicação (artigo), cuja redação final é fruto de um esforço de síntese acurada, em que, dentre as prioridades, se destacam a exposição do encaminhamento metodológico e os principais resultados da investigação.

Uma singularidade importante evidenciada em diversos estudos do Corpus 1 é a escassez de problematização das escolhas teóricas, muitas vezes associada a um (ou mais) instrumentos de coleta de dados. Esse fato permeia os polos epistemológico, teórico e técnico e pode evidenciar inconsistências na articulação das análises (polo morfológico). Como casos em que a IP de PEM é considerada como uma construção complexa e dinâmica, mas os instrumentos de coleta de dados não permitem (nem possibilitam assumir) identificar esse movimento, pois são de natureza estanque, fixos, utilizados em (apenas) um dado momento da investigação. O que queremos frisar é que a natureza do(s) instrumento(s) pode comprometer o processo analítico.

\section{Aspectos caracterizadores das perspectivas epistemológicas de IP de PEM}

No Quadro 2, relacionamos os corpora às perspectivas epistemológicas do conceito de IP de PEM que identificamos e os estudos integrantes do agrupamento proposto. 


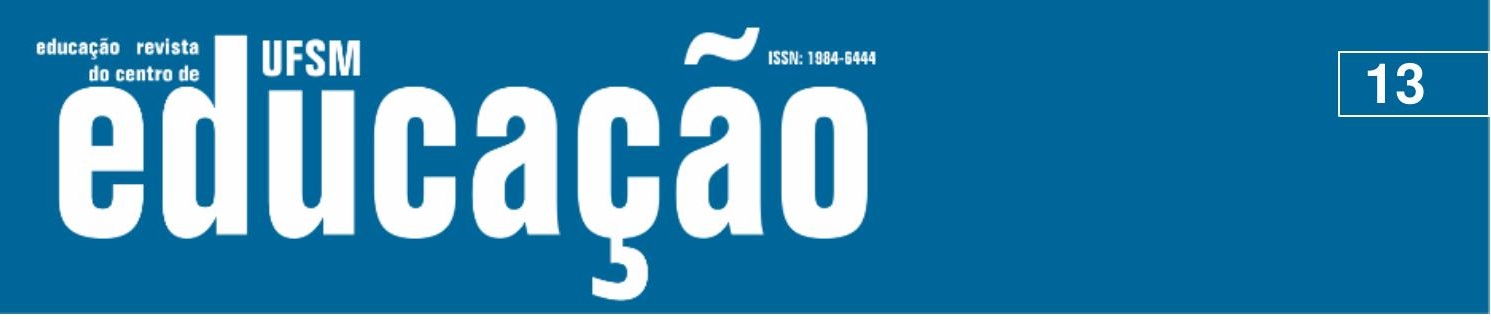

ISSN: 1984-6444 | http://dx.doi.org/10.5902/1984644434406

esses sistemas culturais.

$\checkmark$ Perspectiva generalista - Autores de Referência: não há destaques.

Aspectos característicos: relacionam diversos elementos (saberes, conhecimentos, práticas, experiências) no contexto de formação de professores com a IP: os saberes docentes, a autonomia, a profissionalidade e o desenvolvimento profissional são apontados como fatores influentes nos processos de constituição da IP.

$\checkmark$ Perspectiva holística - Autores de Referência: não há destaques.

Aspectos característicos: envolvem/destacam aspectos afetivos e experienciais (positivos ou negativos), abrangendo acontecimentos do passado, do presente e das expectativas de futuro dos PEM; a resiliência, a confiança, a paixão, as ideologias, a criatividade, as (in)coerências do exercício da profissão, a criticidade e a sensibilidade.

$\checkmark$ Perspectiva pedagógica dos estágios - Autores de Referência: não há.

Aspectos característicos: evidenciam possibilidades de problematizar experiências, de promover aos futuros PEM oportunidades de ações transformadoras de suas expectativas, crenças e concepções. Refletem sobre os anseios em relação ao futuro exercício da profissão, os questionamentos ligados aos conhecimentos, à matemática e a seus processos de ensino e de aprendizagem, o reconhecimento profissional de seus pares e o debate dos aspectos colaborativos.

$\checkmark$ Perspectiva Político-reformista - Autores de Referência: não há.

Aspectos característicos: apresentam estudos em que a análise da IP de PEM abrange as relações de poder, a democracia, as políticas públicas (e as suas diversas formas de implementação), a visão social da profissão. Esses macros aspectos destacados associam-se à formação de professores, sobretudo ao problematizarem o contexto das reformas e a influência de aspectos políticos no processo de formação inicial e continuada de PEM.

$\checkmark$ Perspectiva psicológica/psicanalítica - Autores de Referência: Antônio da Costa Ciampa, Erik Erikson ou Sigmund Freud.

Aspectos característicos: focam nas influências da psique da identidade individual e suas relações (conscientes ou inconscientes) na constituição da IP. A escolha da profissão, a idealização da profissão (e do profissional), a busca por inspirações e os desejos para o exercício da profissão. 


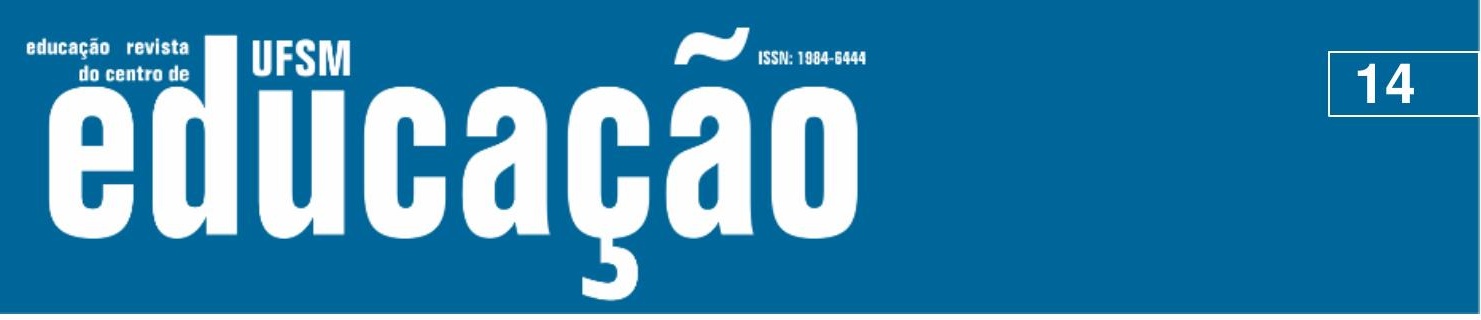

ISSN: 1984-6444 | http://dx.doi.org/10.5902/1984644434406

$\checkmark$ Perspectiva sociológica - Autores de Referência: Claude Dubar e Zygmunt Bauman.

Aspectos característicos: apresentam condicionantes sociais do contexto de trabalho docente no processo de constituição da IP dos PEM, como a visão social da profissão, as relações (e condições) de trabalho, a dinâmica das inter-relações entre as identidades individual, social e profissional.

$\checkmark$ Perspectiva wengeriana - Autores de Referência: Jean Lave e Etienne Wenger.

Aspectos característicos: concentram estudos fortemente influenciados pela Teoria das Comunidades de Prática ( $\mathrm{CoP}$ ), destacando as intencionalidades de fomentar ações de formação docente como iniciativas promotoras do movimento de constituição da IP de PEM.

Por serem representativas de uma gama de aspectos, as perspectivas epistemológicas de IP de PEM identificadas nos levam a defender a problematização da IP como uma perspectiva formativa para os (futuros) professores que ensinam matemática. Refletir a respeito da IP de PEM, dessa forma, significa compreender a IP como uma perspectiva de formação de PEM, de modo que seus aspectos (CDTE) sejam efetivamente considerados em programas e ações formativas, nos quais PEM estejam envolvidos.

Ponderamos que essa proposta de encaminhamento é promissora, pois esses aspectos (CDTE) se inter-relacionam com os aspectos característicos das perspectivas epistemológicas que construímos - cultural, generalista, sociológica, psicológica/psicanalítica, holística, pedagógica dos estágios, político-reformista e wengeriana - e com os aspectos do contexto elencados nesse artigo.

\section{Avanços, (in)certezas e vislumbres das cenas dos próximos capítulos}

No trilhar da identificação dos aspectos caracterizadores do processo de constituição da IP (CDTE) e dos/nos contextos e perspectivas epistemológicas da IP de PEM, alguns apontamentos precisam ser problematizados. 


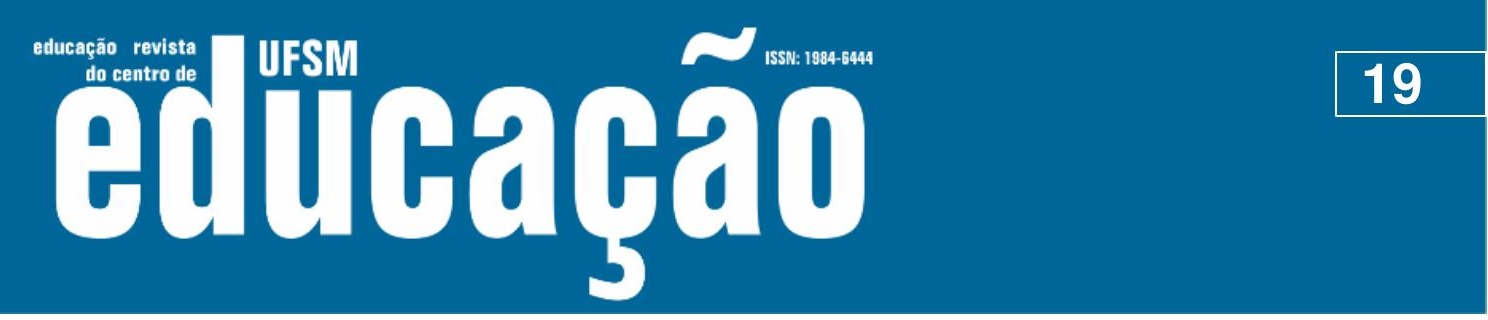

ISSN: 1984-6444 | http://dx.doi.org/10.5902/1984644434406

\section{Referências}

ANDRÉ, Marli Eliza Dalmazo Afonso de André. Pesquisas sobre formação de professores: tensões e perspectivas. In: FONTOURA, H.A.; SILVA, M. (Org.). Formação de professores, culturas: desafios à pós-graduação em Educação em suas múltiplas dimensões. Rio de Janeiro: ANPEd Nacional, p. 24-36, 2011.

BARBOSA, Jonei Cerqueira. Formatos insubordinados de dissertações e teses na Educação Matemática. In: D'AMBRÓSIO, B. S.; LOPES, C. E. (Org.). Vertentes da subversão na produção científica em Educação Matemática. 1. ed., v. 1. Campinas: Mercado de Letras, p. 347-367, 2015.

BEIJAARD, Douwe; MEIJER, Paulien. C.; VERLOOP, Nico. Reconsidering research on teachers' professional identity. Teaching and Teacher Education, Philadelphia: Elsevier, v. 20, p. 107-128, 2004. doi:10.1016/j.tate.2003.07.001

BOOTE, David. N.; BEILE, Penny. Scholars before researchers: on the centrality of the dissertation literature review in research preparation. Educational Researcher, Flórida, v. 34, n. 6, p. 3-15, Aug./Sep. 2005.

CYRINO, Márcia Cristina de Costa Trindade. Preparação e emancipação profissional na formação inicial do professor de matemática. In: NACARATO, Adair Mendes; PAIVA, Maria Auxiliadora Vilela. (Org.). A formação do professor que ensina matemática: perspectivas e pesquisas. Belo Horizonte: Autêntica, p. 77-86, 2006.

CYRINO, Márcia Cristina de Costa Trindade. Mathematics teachers' professional identity development in communities of practice: reifications of proportional reasoning teaching. Bolema, Rio Claro, v. 30, n. 54, p. 165-187, abr. 2016a. doi: 10.1590/1980$4415 v 30 n 54 a 08$

CYRINO, Márcia Cristina de Costa Trindade. Potencialidades da exploração de um caso multimídia como elemento da prática na formação inicial de professores de Matemática. Educação Matemática em Revista, São Paulo, v. 39b, p. 80-89, 2016b.

CYRINO, Márcia Cristina de Costa Trindade. Teacher professional identity construction in pre-service mathematics teacher education: analysing a multimedia case. Proceedings of the 13th International Congress on Mathematical Education. Hamburg: ICME 13, 2016c.

CYRINO, Márcia Cristina de Costa Trindade. Recurso multimídia para a formação de professores que ensinam matemática: elaboração e perspectivas. 1. ed. Londrina: EDUEL: Editora da Universidade Estadual de Londrina, 2016d.

CYRINO, Márcia Cristina de Costa Trindade. Identidade profissional de (futuros) professores que ensinam Matemática. Perspectivas da Educação Matemática, Mato Grosso do Sul, v. 10, p. 699-712, 2017. 


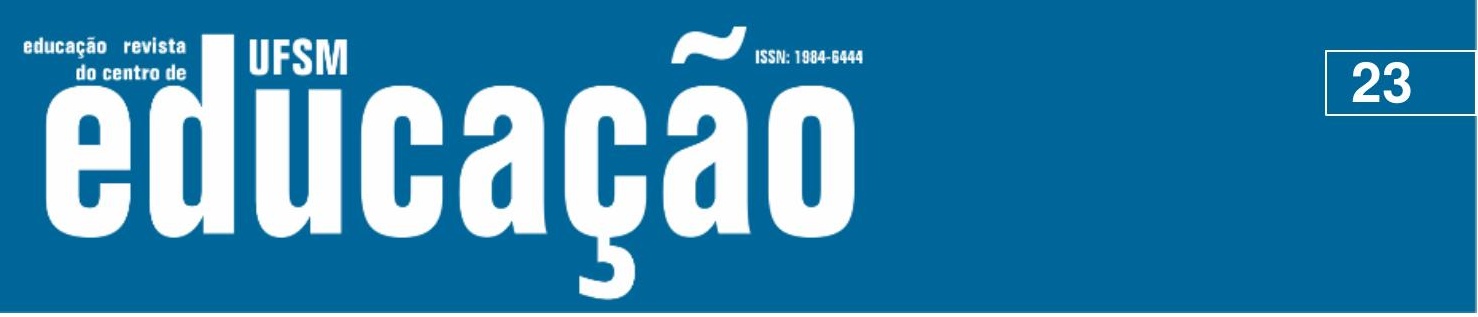

ISSN: 1984-6444 | http://dx.doi.org/10.5902/1984644434406

\section{Referências do Corpus 1}

BATISTA NETO, José Joaquim. O professor de Matemática enquanto sujeito e sua relação com a cultura. 2007. 179 f. Dissertação (Mestrado em Ensino de Ciências e Matemática) - Universidade Cruzeiro do Sul, São Paulo, 2007.

BARBATO, Christiane Novo. A constituição profissional de formadores de professores de matemática. 2016. 322 f. Tese (Doutorado em Educação) Universidade São Francisco, Itatiba, 2016.

BELINE, Willian. Formação de professores de matemática em comunidades de prática: um estudo sobre identidades. 2012. $321 \mathrm{f}$. Tese (Doutorado em Ensino de Ciências e Educação Matemática) - Universidade Estadual de Londrina, Londrina, 2012.

BERANGER, Maurício. Profissionalidade e identidade profissional do professor de matemática: o fenômeno do mal-estar docente e suas implicações. 2007.110p. Dissertação (Mestrado Profissional em Ensino de Matemática) -. Pontifícia Universidade Católica de São Paulo, São Paulo, 2007.

BETERELI, Kelly Cristina. As potencialidades da parceria universidade-escola para professores dos anos iniciais do Ensino Fundamental: reflexões sobre as práticas de aprender e ensinar. 2013.168 f. Dissertação (Mestrado em Educação) Universidade São Francisco, Itatiba, 2013.

BIERHALZ, Crisna Daniela Krause. Curso de Licenciatura em Matemática a distância: o entrelaçar dos fios na (re)construção do ser professor. 2012. 182 f. Tese (Doutorado em Educação) - Pontifícia Universidade Católica do Rio Grande do Sul, Porto Alegre, 2012.

CHAUVET, Márcia Barra Milhomens. Identidade docente e suas implicações no trabalho pedagógico com adolescentes em situação de risco: uma leitura psicanalítica. 2008. 156 p. Dissertação (Mestrado em Educação) - Faculdade de Educação, Universidade de Brasília, Brasília, 2008.

GARCIA, Tânia Marli Rocha. Identidade profissional de professores de Matemática em uma comunidade de prática. 2014. 164 f. Tese (Doutorado em Ensino de Ciências e Educação Matemática) - Universidade Estadual de Londrina, Londrina, 2014.

GUIDINI, Siane Aparecida. O futuro professor de matemática e o processo de identificação com a profissão docente: estudo sobre as contribuições da prática como componente curricular. 2010. 126 f. Dissertação (Mestrado Profissional em Ensino de Matemática) - Pontifícia Universidade Católica de São Paulo, São Paulo. 2010.

JUNQUEIRA, Sonia Maria da Silva. Constituição da identidade de cursos de licenciatura em matemática à luz da modernidade líquida. 2010. 146 p. 


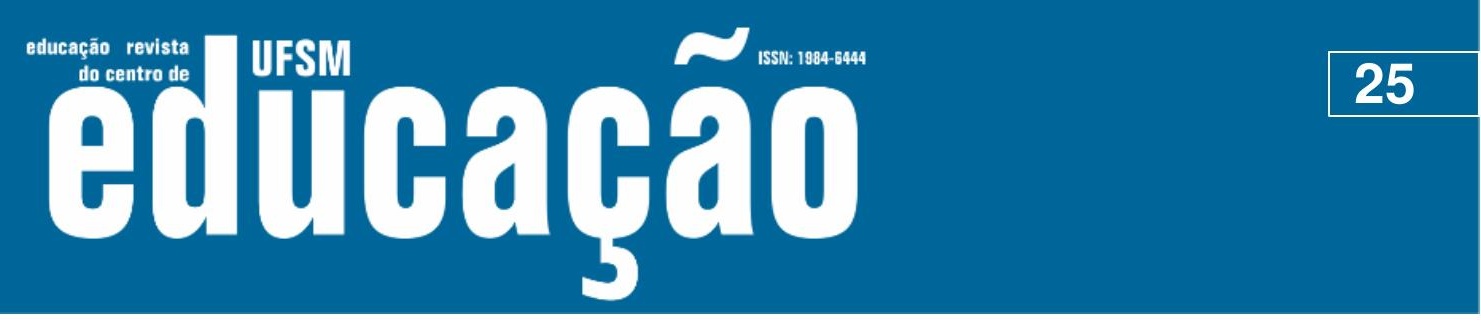

ISSN: 1984-6444 | http://dx.doi.org/10.5902/1984644434406

SOUSA, Josimar de. A construção da Identidade Profissional do professor de matemática no projeto de licenciaturas parceladas da UNEMAT/MT. 2009. 284p. Tese (Doutorado em Educação Matemática) - Pontifícia Universidade Católica de São Paulo, São Paulo, 2009.

SOUZA, Janderson Vieira de. A identidade profissional do professor de Matemática frente aos ciclos de formação e desenvolvimento humano do município de Goiânia à luz da etnomatemática. 2009. 137. Dissertação. (Mestrado em Educação em Ciências e Matemática) - Universidade Federal de Goiás, Goiânia, 2009.

TEIXEIRA, Bruno Rodrigo. O estágio supervisionado e o desenvolvimento profissional de futuros professores de matemática: uma análise a respeito da identidade profissional docente. 2013. 184 f. Tese (Doutorado em Ensino de Ciências e Educação Matemática) - Universidade Estadual de Londrina, Londrina, 2013.

VIEIRA, Andrea Cristina. Um estudo sobre as contribuições do PIBID-FURB para a formação inicial de professores de matemática. 2014. 99 f. Dissertação (Mestrado Profissional em Ensino de Ciências Naturais e Matemática) - Universidade Regional de Blumenau, 2014.

ZANINI, Renato. A identidade profissional do professor de Matemática frente ao trabalho docente no ensino fundamental em ciclos. 2006. 156p. Dissertação (Mestrado em Educação Matemática) - Pontifícia Universidade Católica de São Paulo, São Paulo, 2006.

\section{Referências do Corpus 2}

BENNISON, Anne. Developing an analytic lens for investigating identity as an embedder-of-numeracy. Mathematics Education Research Journal, v. 27, n. 1, p. 1-19, 2015.

BJULAND, Raymond; CESTARI, Maria Luiza; BORGERSEN, Hans Erik. Professional mathematics teacher identity: Analysis of reflective narratives from discourses and activities. Journal of Mathematics Teacher Education, New York, v. 15, n.5, p. 405424, 2012.

BOYLAN, Mark. It's getting me thinking and I'm an old cynic: Exploring the relational dynamics of mathematics teacher change. Journal of Mathematics Teacher Education, New York, v.13, p. 383-395, 2010.

BROWN, Tony; HEYWOOD, David; SOLOMON, Yvette.; ZAGORIANAKOS, Andonis. Experiencing the space we share: Rethinking subjectivity and objectivity. ZDM Mathematics Education, New York, n. 45, 561-572, 2013. 


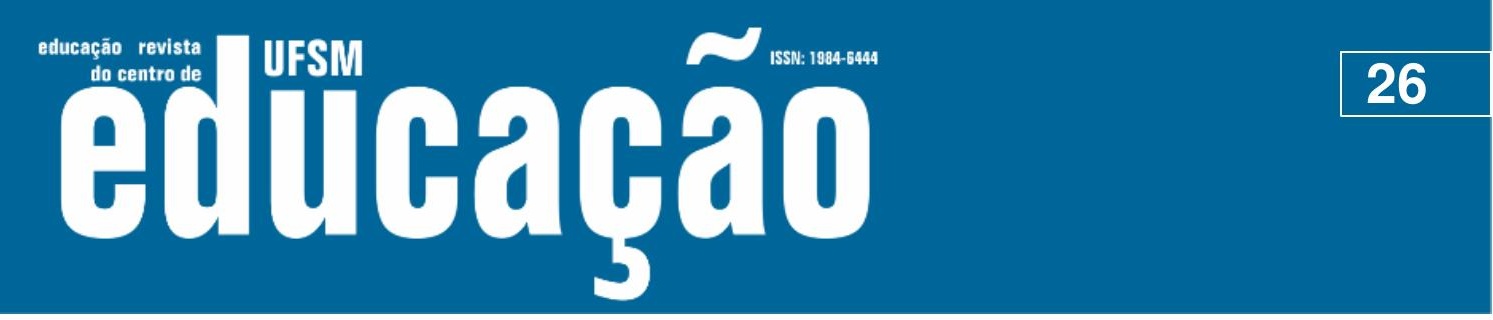

ISSN: 1984-6444 | http://dx.doi.org/10.5902/1984644434406

CYRINO, Márcia Cristina de Costa Trindade. Mathematics teachers' professional identity development in communities of practice: Reifications of proportional reasoning teaching. Bolema, Rio Claro-SP, v. 30, n. 54, p. 165-187, April $2016 a$.

GELLERT, Uwe.; ESPINOZA, Lorena.; BARBÉ, Joaquim. Being a mathematics teacher in times of reform. ZDM Mathematics Education, New York, n. 45, p. 535545, 2013.

GONÇALVES COSTA, Wanderleya Nara; PAMPLONA, Admur Severino. Entrecruzando fronteiras: a educação estatística na formação de professores de matemática. Bolema, Rio Claro, SP, v. 24, n.40, p. 897-911, 2011.

GONÇALVES, Marcos Antônio; DE CARVALHO, Dione Lucchesi. Perscrutando diários de aulas e produzindo narrativas sobre a disciplina estágio supervisionado de um curso de licenciatura em matemática. Bolema, Rio Claro, SP, v. 28, n.49, p. 777798, 2014.

GOOS, Merrilyn E.; BENNISON, Anne. Developing a communal identity as beginning teachers of mathematics: Emergence of an online community of practice. Journal of Mathematics Teacher Education, New York, n. 11, p. 41-60, 2008.

HOBBS, Linda. Examining the aesthetic dimensions of teaching: Relationships between teacher knowledge, identity and passion. Teaching and Teacher Education, Philadelphia: Elsevier, v. 28, n.5, p. 718-727, 2012.

HODGEN, Jeremy.; ASKEW, Mike. Emotion, identity and teacher learning: Becoming a primary mathematics teacher. Oxford Review of Education, Oxford, v. 33, n.4, p. 469-48, 2007.

HOSSAIN, Sarmin; MENDRICK, Heather; ADLER, Jill. Troubling "understanding mathematics in-depth": Its role in the identity work of student-teachers in England. Educational Studies in Mathematics, New York, n. 84, p. 32-48, 2013.

KAASILA, Raimo; HANNULA, Markku S.; LAINE, Anu. My personal relationship towards mathematics has necessarily not changed but... analyzing preservice teachers' mathematical identity talk. International Journal of Science and Mathematics Education, Taiwan. 10, n.4, p. 975-995, 2012.

LLOYD, Gwendolyn M. Preservice teachers' stories of mathematics classrooms: Explorations of practice through fictional accounts. Educational Studies in Mathematics, New York, v. 63, n.1, p. 57-87, 2006.

LUTOVAC, Sonja.; KAASILA, Raimo. Pre-service teachers' future-oriented mathematical identity work. Educational Studies in Mathematics, New York, v. 85, n.1, p. 129-142, 2014. 


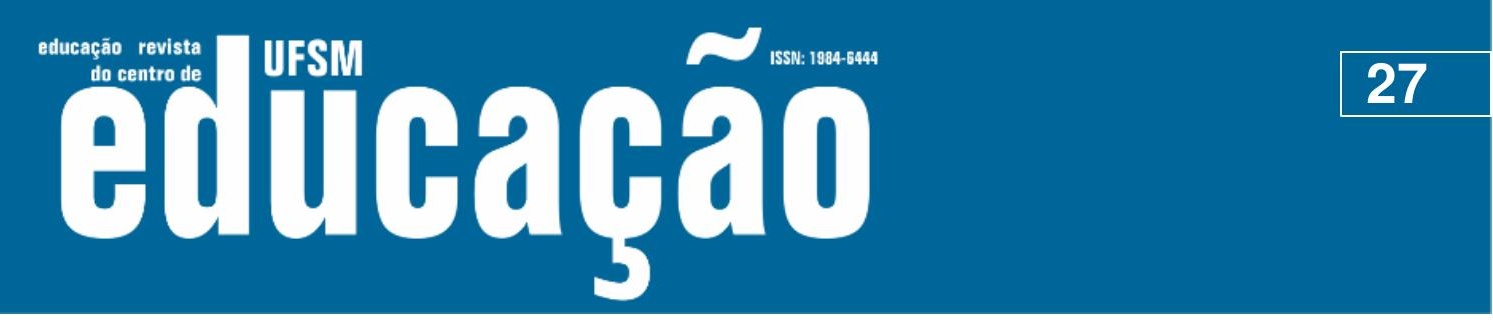

ISSN: 1984-6444 | http://dx.doi.org/10.5902/1984644434406

NOLAN, Kathleen. Dispositions in the field: Viewing mathematics teacher education through the lens of Bourdieu's social field theory. Educational Studies in Mathematics, New York, n. 80, p. 201-215, 2012.

OWENS, Kay. Identity as a mathematical thinker. Mathematics Teacher Education and Development, n. 9, p. 36-50, 2008.

PAZ, Mônica Lana da; FRADE, Cristina. A história de Nair: a força da identidade institucional para a permanência na docência em matemática. Bolema, Rio Claro-SP, v. 30, n.56, p. 1260-1279, dezembro de 2016.

SANHUEZA, Susan.; PENALVA, María Carmen; FRIZ, Miguel. Identidades y competencias profesionales de estudiantes para maestro de educación infantil relativas a la enseñanza de la geometría. Relime, v. 16, n.1, p. 99-122, marzo 2013.

SKOG, Kicki.; ANDERSSON, Annica. Exploring positioning as an analytical tool for understanding becoming mathematics teachers' identities. Mathematics Education Research Journal, v. 27, n.1, p. 65-82, 2015.

TEIXEIRA, Bruno Rodrigo; CYRINO, Márcia Cristina de Costa Trindade. Desenvolvimento da identidade profissional de futuros professores de matemática no âmbito da orientação de estágio. Bolema, Rio Claro-SP, v. 29, n. 52, p. 658-680, 2015.

TICKNOR, Cindy S. Situated learning in an abstract algebra classroom. Educational Studies in Mathematics, New York, n. 81, p. 307-323, 2012.

VAN PUTTEN, Sonja.; STOLS, Gerrit; HOWIE, Sarah. Do prospective mathematics teachers teach who they say they are? Journal of Mathematics Teacher Education, New York, n. 17, p. 369-392, 2014.

\section{Correspondência}

Enio Freire de Paula - Instituto Federal de Educação, Ciência e Tecnologia de São Paulo - Rua José Ramos Júnior, 27-50, CEP 19470000, Jardim Tropical, Presidente Epitácio, São Paulo, Brasil.

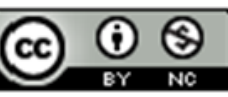

This work is licensed under a Creative Commons Attribution-NonCommercial 4.0 International (CC BY-NC 4.0) 


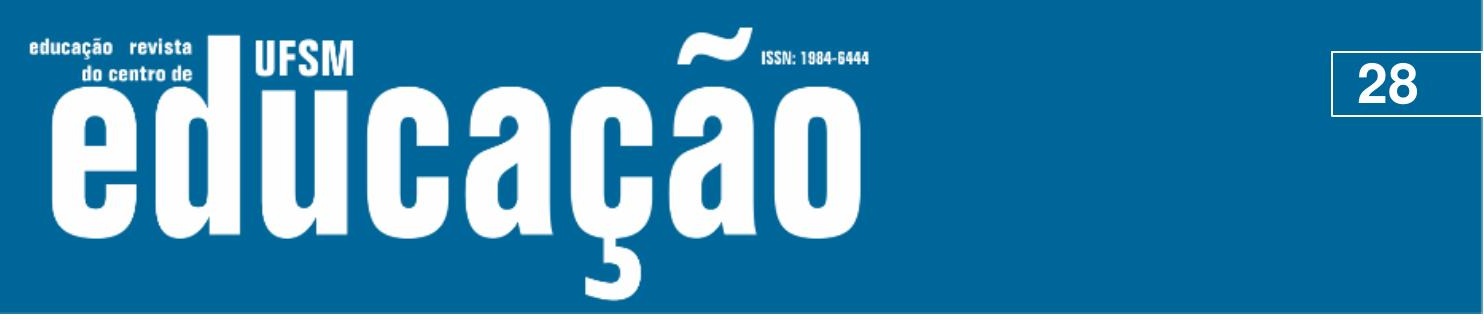

ISSN: 1984-6444 | http://dx.doi.org/10.5902/1984644434406

\title{
Notas
}

\begin{abstract}
${ }^{1}$ Criado em 2003 na Universidade Estadual de Londrina e coordenado pela Prof.a Dra. Márcia Cristina de Costa Trindade Cyrino, o Gepefopem tem investigado perspectivas de formação inicial e continuada na busca de identificar fatores intervenientes no processo de constituição da identidade profissional de PEM em diversos contextos, entre eles as Comunidades de Prática (CoPs).
\end{abstract}

${ }^{2}$ Essa relação foi elaborada, tendo em vista a sequência temporal das citações.

${ }^{3}$ Consideramos nessa investigação como professor que ensina matemática aqueles docentes com formação específica na área da Matemática (licenciados, futuros professores de matemática ou bacharéis), na área de Pedagogia (ou formação para o exercício do magistério) e em áreas afins (como as Engenharias, por exemplo), atuantes no ensino de Matemática em todos os níveis de ensino (da Educação Infantil ao Ensino Superior), seja na modalidade presencial ou a distância.

${ }^{4}$ Educational Studies in Mathematics, International Journal of Science and Mathematics Education, Journal for Research in Mathematics Education, Journal of Mathematics Teacher Education, Mathematics Education Research Journal, Mathematical Thinking and Learning, Mathematics Teacher Education and Development, The Journal of mathematical behavior e ZDM Mathematics Education, Boletim de Educação Matemática (BOLEMA), e a Revista Latinoamericana de Investigación en Matemática Educativa - RELIME.

${ }^{5} \mathrm{Na}$ décima segunda Conferência Internacional sobre Literatura Cinzenta realizada em Praga, em 2010, foi proposta uma nova definição para este termo: “A literatura cinzenta representa vários tipos de documentos produzidos em todos níveis de governo, acadêmicos, empresas e indústria em impressão e formatos eletrônicos protegidos por direitos de propriedade intelectual, de qualidade suficiente para ser coletada e preservada por explorações de biblioteca ou repositórios institucionais, mas não controlados por editores comerciais, ou seja, onde a publicação não é a principal atividade do organismo produtor" (SHÖPFEL, 2010, p. 24).

6 “Compreendemos ponto de enfoque como uma combinação entre os objetivos e as temáticas investigativas assumidas pelos autores dos estudos integrantes do corpus, relacionadas diretamente ao(s) cenário(s) em que as discussões a respeito da identidade profissional foram constituídas” (DE PAULA, CYRINO, 2017).

${ }^{7}$ Ginzburg (1989) defende o paradigma indiciário como capaz de iluminar o objeto/fenômeno em questão e, por meio do raciocínio inferencial e relacional entre as pistas, possibilitar o encontro de indícios ou sinais não perceptíveis de imediato, fato esse que propicia ao investigador incorporar novas hipóteses e conjecturas a respeito de seu foco investigativo.

${ }^{8}$ A partir desse momento no texto, para nos referimos conjuntamente a esses quatro aspectos caracterizadores, utilizaremos a sigla CDTE.

9 "Não a vulnerabilidade que enfraquece, susceptibiliza e é paralisante [...], mas a que nos permite suspender por alguns instantes, mais ou menos longos, e mais ou menos frequentes, as nossas certezas e convicções. Aquela que nos faz questionar a nós próprios. Também a vulnerabilidade no sentido de nos expormos aos outros e, como tal, podermos tornar-nos 'alvo de crítica, de contestação'. Do nosso ponto de vista, por vezes, o que julgamos ser uma incapacidade do futuro professor para reflectir sobre as suas aprendizagens e sobre a sua prática, no contexto da prática de ensino supervisionada, pode ter a ver com o facto de que essa reflexão depende de condições pessoais, tais como a confiança e a vontade de assumir riscos" (OLIVEIRA; CYRINO, 2011, p. 112, grifo nosso).

${ }^{10}$ Optamos pelo uso do termo em inglês (multipaper) para nos atermos, nesse caso, a uma tese construída e organizada como uma coletânea de vários artigos interconectados a uma questão de investigação.

${ }^{11}$ Os trabalhos de Oliveira (2010) do Programa de Pós-Graduação em Ensino, Filosofia e História das Ciências da UFBA/UEFS; Estevam (2015) do Programa de Pós-Graduação em Ensino de Ciências e Educação Matemática da UEL, Dantas (2016) do Programa de Pós-Graduação em Educação Matemática da UNESP/RC; Santana (2017) e Januário (2017) do Programa de Pós Graduação em Educação Matemática da PUC/SP e Lautenschlager (2017) do Programa de Pós-Graduação em Ensino e História das Ciências e da Matemática da UFABC, exemplificam essa multiplicidade/diversidade de formas dentro da perspectiva do modelo multipaper. 


\section{Dism

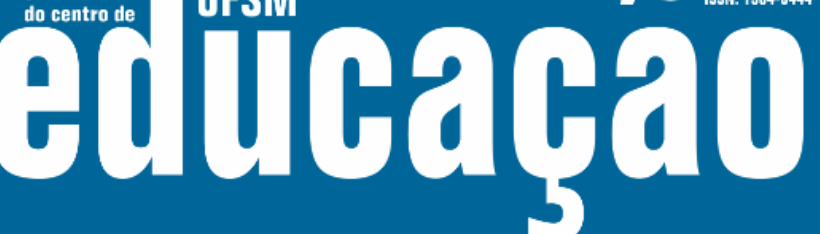

ISSN: 1984-6444 | http://dx.doi.org/10.5902/1984644434406

${ }^{12}$ Essa é uma denúncia acadêmica importante. Posto que grande parte dos autores integrantes do corpus nacional não esteve presente no corpus dos artigos, isso indica que os resultados oriundos das investigações em dissertações (de mestrado acadêmico e profissional) e teses não foram publicados (pelo menos no intervalo analisado) na forma de artigos. 